\title{
BINOCULAR FIXATION FOR SCOTOMETRY AT ONE METRE
}

\author{
BY \\ J. Foster \\ LEEDS
}

Scotometry at $33 \mathrm{~cm}$. while recognised as less accurate, and as being a less delicate test than at one or two metres, possessed for a long time the compensating advantages of binocular fixation and rapid recording. In the last few years the speed of recording at greater distances than this has been increased by various devices. Amongst the latter one may mention Tudor Thomas' projection device, Affleck Greeves' stitched screen, and the Foster protractor.

Binocular fixation at longer distances is still rather a problem, however, and existing apparatus is either apt to interfere with the field of the examined eye, to be somewhat inaccurate, or complicated and expensive.

Cowan and Markov (Amer. Jl. Ophthal., 1929, p. 656) have produced a simple method which overcomes these difficulties, but their device is still rather unwieldy.

The device described below incorporates portions of the design of several existing methods of binocular fixation. It allows for a long working distance, non-interference with the field of the tested eye, a simple check on the accuracy of fixation, and is relatively simple in practice and construction.

Two models are described; the first being more suitable where illumination on the scotoma screen is variable, and where a chin rest (to which the apparatus can be fastened) is employed.

The second model (which is the one I employ personally) is more convenient where a chin rest is not used, and the illumination of the Bjerrum screen a constant quantity. The instrument consists of two parts :

1. Circular Target.-This is a grey circle of 5in. external diameter, painted on a 7 in. square of black paper, which is pinned to the Bjerrum screen so that the centre of the circle overlies and conceals the fixation point on the screen, in the same way as its precursor the Fincham target.

The circle has four inward projections like the stubs of the spokes of a wheel. The screen should have an even surface illumination of at least $7 \mathrm{ft}$. candles-as poor illumination of the target renders it invisible through the second part of the instrument. 
2. The Cross Projector.-This resembles an ordinary ophthalmoscope, with a battery handle containing a resistance.

The patient, seated at one metre, holds the instrument in one hand, and looks through the sight hole through a half-silvered mirror, which is sloping at an angle of $45^{\circ}$ towards, instead of away from, the user, as in an ordinary ophthalmoscope.

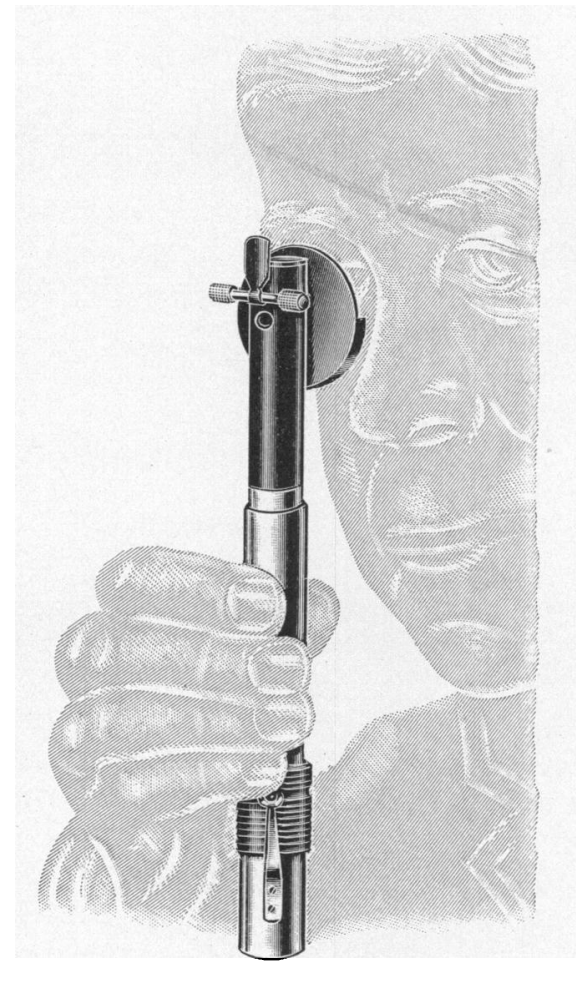

A movement of the switch projects the illuminated image of a cross on to this mirror-which appears as if it were. situated on the Bjerrum screen.

The resemblance to an ophthalmoscope is very strong, and the head of the device can be adapted to any self-illuminated ophthalmoscope.

When it is desired to make a scotometric examination of a patient (at one metre) with a central scotoma in his left eye, it may prove impossible to obtain central fixation for the eye, and the target may be entirely or partially invisible. If he looks through the unilluminated fixation device with the other eye, the whole of the target will be visible. (Fig. 1.) 


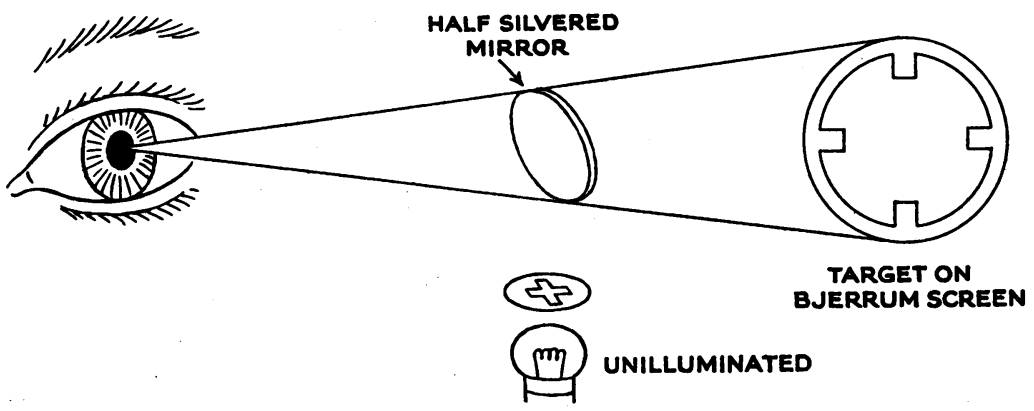

Fig. 1.

The device is now switched on at a low voltage. The target and an illuminated cross are simultaneously visible, and he can then centre the latter to fuse with the inward projections of the former, by moving the battery handle which he holds (Fig. 2) in front of the right eye.

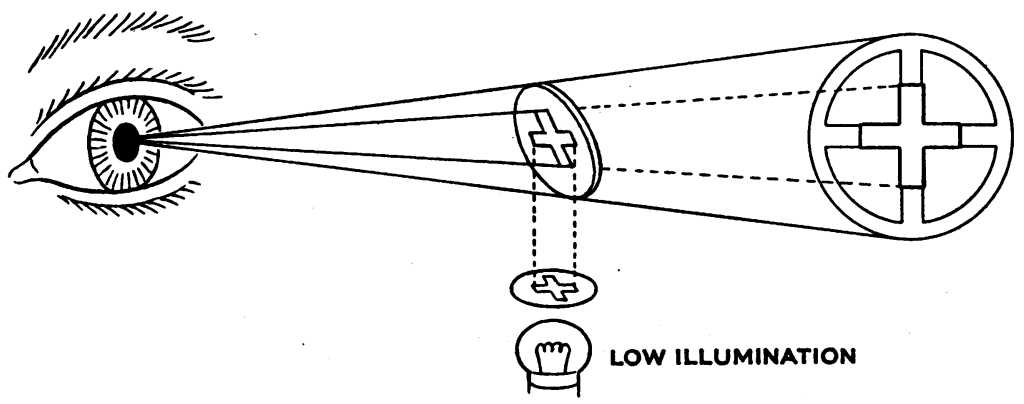

FIG. 2.

$A$. The illumination is increased by turning the switch, and the target gradually disappears from view of the good eye, leaving only the cross, which the patient fixes. (Fig 3.)

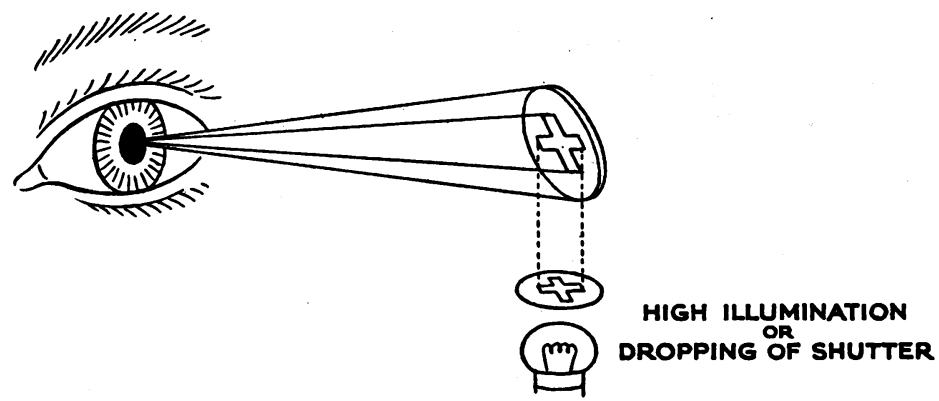

Fig. 3. 
Scotometric examination of the left eye is now carried out, and the fixation checked occasionally by reducing the illumination in the fixation device to make sure that the cross is still centred in the target.

$B$. If enough of the target is still visible to the bad eye to allow of fusion symmetrically with the cross, stages one and two may be omitted.

If a normal person centres the cross by method " A " with his right eye, and keeps the other closed, the cross will appear to shift a little horizontally from the centre of the circle when the left eye is re-opened. Similarly, if the fully illuminated cross is fused with the target, and illumination then reduced, it will be found to be displaced in the opposite direction. This phenomenon is due to heterophoria, and when it can be estimated, as in a normal person, a compensating trial case prism can be placed in the groove behind the sight hole of the instrument.

As, however, in actual practice, central fixation is completely lost in most of the cases one wishes to test, and the heterophoria probably does not vary throughout the examination, this slight error can be neglected.

\section{Model II}

I, personally, do not employ a chin rest for scotometry, and it was found in practice that certain patients held the instrument rather unsteadily, so that moving the resistance moved the instrument a little. If, therefore, one has a constant illumination on a scotoma screen, and the device is not fixed to a chin rest or made firm in any other way, another modification of it may prove more convenient in use, and it is in this form that the device was finally made up.

The resistance is replaced by a switch, and the silvering on the mirror is removed so that at maximum illumination it is still possible to see the circle and the cross simultaneously with the good eye. A small shutter is dropped over the sighting hole between the screen and the silvered glass, producing the same effect as increasing the illumination. The shutter can be raised and lowered very easily from either side of the patient to check whether the cross is still in the circle or not.

The diagnostic devices described in this article are all produced by Messrs. Rayner and Co., 100, New Bond Street, London, W.1, for whose assistance in their development I am greatly obliged. 